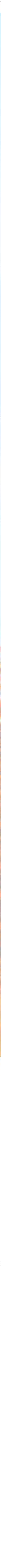

DAS REIZDARMSYNDROM (RDS, Colon irritabile) gehört zu den häufigsten Erkrankungen in Deutschland. Bis zu 20\% der Bevölkerung und somit etwa 16 Millionen Menschen sind Schätzungen zufolge betroffen - Frauen doppelt so häufig wie Männer. Angesichts dieser Zahlen mag es erstaunen, dass die Wissenschaft immer noch nach den Ursachen dieser Erkrankung sucht. Dabei ist der Forschungsbedarf enorm, schon wegen der hohen Kosten für die umfangreiche Diagnostik - vor allem aber wegen des hohen Leidensdrucks bei Betroffenen.

\section{Alte neue Erklärungsmodelle:}

\section{Temperamenten- und Ernährungslehre}

Fragen der Verdauung beschäftigten bereits die Humoralmedizin der Antike und des Mittelalters. Nach deren Konzept entscheidet vor allem das Mischungsverhältnis der vier Kardinalsäfte über die Entstehung von Krankheiten. Man ging davon aus, dass jeder Mensch von Geburt an ein bestimmtes Temperament (siehe S. 16) ausprägt und damit in ihm natürlicherweise einer der Körpersäfte (Humores) vorherrscht. Durch die Lebensakti- 
vitäten verbrauchte Humores werden hierbei über Nahrung und Verdauung wieder aufgefüllt. Aus dieser Perspektive erschließt sich die Bedeutung der Diätetik im Mittelalter: Machte man mit der Ernährung etwas falsch oder funktionierte die Kochung (Verdauung) nicht richtig, musste das unweigerlich zu Erkrankungen führen.

Seit wir Krankheitserreger wie Viren und Bakterien kennen, hat sich unsere Einsicht in Krankheitsentstehungsprozesse grundlegend geändert. Anders als die Mediziner des Mittelalters können wir mit bildgebenden Verfahren in Magen und Darm hineinschauen. Histologische und molekulargenetische Untersuchungsmethoden liefern uns zusätzlich eine Fülle von Informationen. Aus der Sicht des Reizdarmpatienten helfen diese Innovationen allerdings wenig: Die moderne Medizin kann ihm sagen, welche möglichen Ursachen bei ihm nicht vorliegen: Das Reizdarmsyndrom ist eine Ausschlussdiagnose. Mit der tröstlichen Auskunft, ,nichts Schlimmes“ zu haben, werden die Patienten dann häufig alleingelassen, ohne dass ihnen eine Therapie vorgeschlagen oder gar eine Präventionsstrategie an die Hand gegeben wird. Zu komplex und individuell verschieden ist die Pathogenese dieser Beschwerden. Da wir somit auf die Notwendigkeit einer Symptombekämpfung zurückgeworfen werden, sind die traditionellen Heilanwendungen bei Darmbeschwerden nicht weniger aktuell als vor Jahrhunderten. Eine Typisierung des Patienten im Sinne der Humoralpathologie kann hierbei wertvolle Hinweise zur Beratung der Patienten liefern (siehe „Falle Sensibilität: Melancholiker als Mustertyp der Reizdarmpatienten“).

\section{Colon irritabile als Ausschlussdiagnose nach Diagnostik-Odyssee}

Beim Reizdarmsyndrom stehen vier Symptomkomplexe im Vordergrund: Durchfall, Verstopfung, Blähungen und Bauchschmerzen beziehungsweise -krämpfe. Diese treten meist kombiniert auf, zum Beispiel in chronischem Wechsel von Verstopfung und Durchfall. Es gibt eine Vielzahl unterschiedlichster Erkrankungen, die solche Symptome hervorrufen könnten. Alle müssen mit anerkannten diagnostischen Methoden ausgeschlossen werden. Ultraschalluntersuchungen sowie Magen- und Darmspiegelungen gehören hierbei zur Basisdiagnostik zum Ausschluss krankhafter Prozesse im Bauchraum. Laborwerte geben Aufschluss über mögliche Leber-, Galle-, Pankreas- oder Schilddrüsenerkrankungen. Stuhluntersuchungen schließen bakterielle oder parasitäre Infektionen aus. Ein besonders weites Feld ist die Abklärung von Lebensmittelallergien und -intoleranzen.

Da die Chronizität zu den wesentlichen Charakteristika des Reizdarmsyndroms zählt, haben viele Patienten eine lange Reihe ergebnisloser Arztbesuche und Untersuchungen hinter sich. Lange Zeit rechnete man die Störung schlussendlich den psychosomatischen Erkrankungen zu. Den wohlgemeinten ärztlichen Rat, psychotherapeutische Hilfe zu suchen, empfinden jedoch nicht wenige Patienten als kränkend. Angesichts ihrer fort-

\author{
KURZ GEFASST \\ 1 Das Reizdarmsyndrom betrifft fast jeden Fünften, insbesondere \\ den Typus des Melancholikers, und zeigt sich mit Durchfall, \\ Verstopfung, Blähungen und Bauchschmerzen. \\ Eine veränderte Darmsteuerung und -wahrnehmung sowie \\ Störungen des Mikrobioms sind häufig beteiligt, wobei Nah- \\ rungsmittelintoleranzen und andere Magen-Darm-Erkrankungen \\ auszuschließen sind. \\ 3 Gerb- und Quellstoffdrogen wie Eichenrinde oder Flohsamen \\ 3 können gemeinsam mit anderen traditionellen Maßnahmen \\ besonders beim Durchfalltyp deutliche Verbesserungen bringen.
}

dauernden körperlichen Beschwerdesymptomatik fühlen sie sich nicht ernst genommen - zu Recht, wie neuere Untersuchungsergebnisse zeigen: Derzeit wird diskutiert, inwieweit immunologisch bedingte Veränderungen der Nervenübermittlung nach einer durchgemachten Darminfektion am Krankheitsprozess beteiligt sind.

\section{Veränderte Reizwahrnehmung: Verdauung ist Nervensache}

Über 100 Millionen darmeigene Nervenzellen steuern unser Verdauungssystem. Zusammen werden sie im Hinblick auf ihre Komplexität auch als Darmhirn bezeichnet. Dessen Kommunikation mit dem Gehirn scheint bei Reizdarmpatienten verändert zu sein: Sonst normale Reize aus dem Darm, zum Beispiel über seinen Füllungszustand, werden vom Gehirn als Schmerzreize fehlinterpretiert. Der Patient erlebt dies als Überempfindlichkeit seiner Eingeweide. Bei der Untersuchung durch Palpation beispielsweise sind die Patienten deutlich schmerzempfindlicher als Nichtbetroffene. Auch die neuronale Koordination der Darmmuskulatur scheint gestört: Die Darmperistaltik wird manchmal reduziert, manchmal beschleunigt. Daher der häufige Wechsel zwischen Verstopfung und Durchfall.

Auf Stressfaktoren reagiert der Darm sehr sensibel: Die Symptome verschlimmern sich oder neue Schübe werden ausgelöst. Die bei Reizdarmpatienten häufigen Schlafstörungen könnten ebenfalls auf diese reduzierten Reizschwellen bei der Zusammenarbeit von Darm und Gehirn zurückzuführen sein.

\section{Falle Sensibilität: Melancholiker als Mustertyp des Reizdarmpatienten}

In der Humoralmedizin versucht der Therapeut, seine Patienten zu typisieren und auf dieser Grundlage einen Therapieplan auszuarbeiten. Eines der vier Temperamente beschreibt den Reizdarmpatienten fast exakt: der Melancholiker. Was heute gerne unter diesem Typus verstanden wird - eine traurige, trübsinnig an sich selbst leidende Persönlichkeit -, wird ihm allerdings nicht gerecht. Nach der ursprünglichen Definition handelt es sich um einen empfindsamen, nachdenklichen Men- 
schen, mitfühlend und geprägt durch hohe Moralansprüche und großes Gerechtigkeitsempfinden. Eine gute Körperwahrnehmung ist gekoppelt mit erhöhter Sensibilität und Irritabilität. Vieles geht dem Melancholiker nahe oder verursacht ihm Sorgen. Er macht die Erfahrung, dass in seinem Lebensumfeld manches seinen Ansprüchen nicht gerecht wird. Seine weniger sensiblen Mitmenschen können aber seine Reaktionen darauf nicht verstehen und beantworten diese mit Abwehr oder Distanz. In der Folge entwickelt der Melancholiker eine eher pessimistische, ängstliche oder misstrauische Grundhaltung. Die Humoralpathologie sagt Probleme mit Magen und Darm bei diesem Menschentyp voraus. Sie begründet Magenübersäuerungen, Blähungen und Verdauungsprobleme mit einem Überschuss an Melanchole, der Schwarzen Galle, in Magen und Darm.

\section{Bei Nebenwirkungen fragen Sie Ihren Heilpraktiker}

Was kann die Typisierung als Melancholiker zur Behandlung und Beratung von Reizdarmpatienten beitragen? Zum einen sind Melancholiker prädestiniert für die Entwicklung von Nebenwirkungen. Dies ist eine wichtige Erkenntnis für jeden Therapeuten, denn das gilt nicht nur bei der Einnahme chemischsynthetischer Medikamente, sondern ebenso bei pflanzlichen Arzneimitteln oder Probiotika. Zum anderen kann auch eine Ernährungsumstellung, zum Beispiel auf ballaststoffreichere Kost, Probleme bereiten. Diese Schwierigkeiten müssen nicht auf einen Nocebo-Effekt zurückzuführen sein, sondern stellen möglicherweise eine Reaktion des tatsächlich übersensiblen Metabolismus und Nervensystems dar.

In der Praxis bedeutet das, dass besonders viel Wert auf die Medikamentenanamnese gelegt werden sollte. Gerade bei älteren Menschen mit Mehrfachmedikation treten durch Nebenund Wechselwirkungen gehäuft Magen-Darm-Störungen auf. Für die eigene Verordnung von Arzneimitteln hat es sich bewährt, jede neue Behandlung behutsam mit niedrigen Dosen einzuschleichen. Eine gute Möglichkeit ist die Anwendung einer Kinderdosis für die ersten ein oder zwei Wochen und eine fest vereinbarte Rückmeldung durch den Patienten vor einer Steigerung der Dosis. Dies gilt auch für Probiotika im Rahmen einer Darmsanierung, auch wenn nur Bakterienspezies supplementiert werden, die laut Stuhluntersuchung in zu geringem Maß vorhanden sind.

\section{Krankheitsprophylaxe und Entspannung: Schwerpunkt Vorbeugung}

Ein zweiter wichtiger Aspekt ist, anderen - insbesondere antibiotikapflichtigen - Erkrankungen vorzubeugen, die den Darm zusätzlich belasten würden. Eine gute Mundhygiene ist bei Reizdarmpatienten zur Prophylaxe von Zahnerkrankungen erforderlich. Bei Frauen sollte man sich nach der Häufigkeit von
Blasenentzündungen erkundigen und gegebenenfalls präventive Maßnahmen einleiten. Die geringe Stresstoleranz des melancholischen Typus kann zu Magen- und Refluxerkrankungen führen: Häufig treten Reizmagen und Reizdarm gemeinsam auf. Die Vermittlung von Entspannungstechniken, die dem Patienten zusagen, ist daher ein besonders wichtiger Therapiebaustein.

\section{Merke: Häufig treten Reizmagen und Reizdarm gemeinsam auf.}

Wir befinden uns hier als Therapeuten genau genommen in keiner anderen Situation als die Humoralmediziner in der Zeit der Klostermedizin: Wir haben eine Erkrankung vor uns, deren Ursachen wir nicht kennen und die wir daher nur symptombezogen behandeln können. Deshalb liegt ein wesentlicher Schwerpunkt bei der Vorbeugung beziehungsweise Vermeidung von Rückfällen. Ganz im Sinne der Beachtung der Res non naturales (siehe S. 17) kann man die Patienten ganzheitlich unterstützen, indem man diese Faktoren bespricht. Eine ausgedruckte Patienteninformation (siehe S. 28) zum Mitnehmen ist dabei hilfreich.

\section{Diagnostik: Intoleranzen und Mikrobiom untersucht?}

Die überwiegende Mehrzahl der Reizdarmpatienten bringt schon aktuelle Arztberichte zur Ausschlussdiagnose in die Naturheilpraxis mit. Diese umfassen fast immer die bildgebenden Verfahren und die Labordiagnostik. Nahrungsmittelallergien sind meist bekannt. Was die häufigsten Nahrungsmittelintoleranzen, vor allem auf Laktose, Fruktose, Histamin und Prolamine (Gluten/Gliadin) betrifft, wurde jedoch selten konsequent gescreent. Die Erfahrung zeigt, dass diese oft zumindest phasenweise kombiniert auftreten. Lassen Sie daher vor Beginn einer Therapie diese Intoleranzen komplett durch anerkannte Testverfahren abklären. Atemtests für Laktose und Fruktose sowie Antikörpersuchtests auf Gliadin, Transglutaminase (beide als Teil der Zöliakiediagnostik) und Bestimmungen des Histamin- und Diaminoxidasespiegels (Histaminintoleranz) stellen die eigene Therapie auf eine stabile Datenbasis.

Merke: Häufig entpuppt sich der vermeintliche Reiz-
darm als Intoleranz auf Laktose, Fruktose, Histamin
oder Gluten. Lassen Sie diese daher vor Beginn einer
Therapie durch anerkannte Testverfahren abklären.

Stuhltests zum pH-Wert des Darms und der Zusammensetzung der Darmflora geben wichtige ergänzende Hinweise zum Zustand der Mikrobiota. Sie sind unerlässlich für eine auf den Patienten zugeschnittene Darmsanierung durch Prä- und Probiotika, die ja auch in engem Zusammenhang mit den zu erarbeitenden Ernährungsempfehlungen steht. 


\section{Erst helfen, dann testen:}

\section{Beratung zur Symptombehandlung}

Befindet sich der Patient bei der Erstvorstellung in einer akuten Belastungssituation durch Verstopfung oder Durchfall, wird er nicht auf die Auswertung der Tests oder Ernährungsprotokolle warten wollen. Zusätzlich zur Besprechung der Allgemeinmaßnahmen (siehe Patienteninformation, S. 28) kann bei Obstipation empfohlen werden: Täglich morgens als erstes Frühstück 1 EL Leinsamen (zum Beispiel Linusit ${ }^{\circledR}$, Fa. Bergland), 1 EL Haferkleie, und 1 EL Milchzucker mit Joghurt und/oder Apfelmus zu einem Brei verrührt einnehmen. Danach reichlich Tee oder angewärmtes Wasser trinken. Bei regelmäßiger Anwendung stellt sich die Wirkung recht zuverlässig ein.

Eine tägliche Heublumenauflage nach Pfarrer Kneipp wirkt bei Bauchschmerzen und Verstopfung sowie Durchfall lindernd: Ein Heublumensäckchen über Wasserdampf auf etwa $50^{\circ} \mathrm{C}$ erhitzen (zum Beispiel zwei Kochlöffel über den Wassertopf legen und das Säckchen darauf platzieren). Nach Temperaturkontrolle auf den Bauch legen, mit einem Zwischentuch und einer Wolldecke abdecken und entspannt liegen, solange die Temperatur als angenehm empfunden wird. Gegebenenfalls noch eine Bauch-Selbstmassage anschließen.

Reizdarmpatienten, die von chronischen Durchfällen geplagt werden, stehen unter einem besonderen Leidensdruck und wünschen sich rasche Abhilfe. Sie müssen sich in ihrem Sozialleben einschränken und ihre Aktivitäten häufig nach der Reichweite einer Toilette ausrichten. Über die Gefahr von Dehydratation und Elektrolytverlust müssen sie unterrichtet und über Gegenmaßnahmen (siehe Kasten „Trinklösung bei starkem oder
REZEPTUR

\section{Trinklösung bei starkem oder länger anhaltendem Durchfall}

Um bei starkem oder länger anhaltendem Durchfall Verluste an Wasser und Elektrolyten auszugleichen, empfiehlt die WHO eine Salz- und Glukosetrinklösung:

4 TL Zucker (Saccharose), 3/4 TL Kochsalz, 1 Tasse Orangensaft (ersatzweise zur Kaliumzufuhr zwei Bananen dazu essen) und 11 stilles Wasser mischen und verrühren. Die Trinkmenge dieser Lösung sollte etwa $40 \mathrm{ml}$ pro kg Körpergewicht innerhalb von 24 Stunden betragen. Dies entspricht bei einem Körpergewicht von $75 \mathrm{~kg}$ drei Litern pro Tag!

länger anhaltendem Durchfall“) informiert sein. Auch für Hinweise zur Hygiene und Pflege der strapazierten Haut im Analbereich, zum Beispiel durch Sitzbäder mit Kamillen- oder Ringelblumenauszügen, sind Betroffene erfahrungsgemäß dankbar.

\section{Therapiestart mit initialer Entlastungskur}

Bevor die eigentliche Therapie beginnt und bis die Testergebnisse vorliegen, hat sich außerdem das folgende Therapieschema zur Überbrückung der Wartezeit bewährt, wenn gerade kein akuter Krankheitsschub vorliegt.

- TABELLE 1

\section{Heilpflanzen zur Behandlung der funktionellen Diarrhö}

\begin{tabular}{|c|c|c|c|c|}
\hline Wirkstoffe & Drogen & adstringierend, abdichtend & flüssigkeitsbindend & entzündungshemmend \\
\hline \multirow[t]{8}{*}{ Gerbstoffe } & Brombeerblätter & ++ & 0 & 0 \\
\hline & Eichenrinde & ++ & 0 & ++ \\
\hline & Frauenmantelkraut & ++ & 0 & 0 \\
\hline & Gänsefingerkraut & ++ & 0 & + \\
\hline & Heidelbeerfrüchte & ++ & 0 & 0 \\
\hline & Odermennigkraut & ++ & 0 & ++ \\
\hline & Teeblätter, schwarz oder grün & +++ & 0 & 0 \\
\hline & Tormentillwurzelstock (Blutwurz) & +++ & 0 & ++ \\
\hline \multirow[t]{3}{*}{ Quellstoffe } & Flohsamenschalen & 0 & +++ & 0 \\
\hline & Apfelpektin & 0 & ++ & 0 \\
\hline & Karottenpektin (Suppe) & 0 & + & 0 \\
\hline Adsorbenzien & Kaffeekohle & 0 & +++ & 0 \\
\hline
\end{tabular}


Für eine initiale Entlastungskur eignen sich zur Unterstützung von Magen und Leber Ventracid ${ }^{\circledR}$ Tbl. (Fa. Repha) mit Curcuma, Cholin und Verdauungsenzym-Konzentrat, in der ersten Woche $2 \times$ tgl. 1 Tbl., danach $3 \times$ tgl. 2 Tbl. jeweils zu den Mahlzeiten.

Zur Regulation der Verdauung sind Flohsamenschalen das Mittel der Wahl. Sie wirken mild abführend bei Verstopfung, flüssigkeitsbindend bei Durchfall und ernährend für die Darmflora: In der ersten Woche $1 \times$ tgl., danach $2 \times \operatorname{tgl}$. 1 TL (ca. $5 \mathrm{~g}$ ) Flohsamenschalen mit $200 \mathrm{ml}$ Wasser, Tee oder klarer Suppe mischen, umrühren und trinken. Unbedingt ein Glas Wasser hinterhertrinken! Flohsamen dürfen nur mit ausreichend Abstand zu Mahlzeiten und Medikamenteneinnahme eingenommen werden, da Nähr- und Wirkstoffe an sie gebunden werden und dem Körper dann nicht mehr zur Verfügung stehen. Die Entlastungskur dauert mindestens vier Wochen.

\section{Ernährungsprotokoll als Therapiegrundlage}

In diesen ersten Wochen sollte der Patient ein Befindlichkeitsund Stuhltagebuch sowie ein Ernährungsprotokoll führen. Am besten gibt man dazu Formulare mit vorgedruckten Kriterien und Werteskalen zum Beispiel zu Stuhlkonsistenz, Nahrungsmitteln und Wohlbefinden aus, die sich einfach ausfüllen lassen. Die Herausforderung wird sein, diese Daten und alle Testergebnisse miteinander in Beziehung zu setzen, um die indivi- duelle Therapie zu erarbeiten. Gerade beim Melancholiker-Typus sind spürbare Symptomverbesserungen zum Aufbau einer langfristigen vertrauensvollen Patient-Therapeut-Beziehung wichtig. Diese ist ihrerseits Teil der Therapie.

\section{Therapie bei Reizdarm vom Durchfalltypus}

Adstringenzien (von lat. adstringere = zusammenziehen) dichten die Oberfläche der Darmschleimhaut ab und wirken damit sekretionshemmend und blutstillend. Pflanzliche Gerbstoffe wie die Katechin-Gerbstoffe der Eichenrinde (Therapiebeispiel siehe Rezepturen) bewirken dies, indem sie die Oberflächenproteine auf der Schleimhaut miteinander vernetzen. Dioskurides schreibt dazu in seiner Materia Medica. „Die ganze Eiche hat adstringierende und austrocknende Kraft. Am meisten adstringiert aber von ihr die hautartige Substanz zwischen Rinde und Stamm. Die Abkochung davon wird denen gegeben, die am Magen, an Dysenterie leiden." Dysenterie, in mittelalterlichen Texten auch als Bauchfluss bezeichnet, ist eine veraltete Bezeichnung für die Ruhr. Höchstwahrscheinlich waren von der Antike bis in die Neuzeit hinein Durchfälle zumeist auf Infektionen oder Lebensmittelvergiftungen zurückzuführen. Ob es in früheren Epochen ein Reizdarmsyndrom gemäß unserer heutigen Definition gab, ist unbekannt.

\section{Bei Reizdarmsyndrom vom Durchfalltyp (Beispiele)}

\section{Schwarz- oder Grüntee}

1 TL der Blätter pro Tasse in $150 \mathrm{ml}$ Wasser 10 min kochen, abseihen, im Akutstadium alle 2-3 Stunden 1 Tasse ungesüßt trinken. In schweren Fällen durch 30 Tr. einer Blutwurztinktur verstärken. Grüntee wirkt stärker als Schwarztee.

\section{Eichenrinden-Tee}

$3 \mathrm{~g}$ (circa $1 \mathrm{TL}$ ) des Pulvers (zum Beispiel von Bombastus) in $300 \mathrm{ml}$ Wasser geben, aufkochen, 15 min sieden lassen, abseihen und in eine Thermoskanne umfüllen. $2 \times$ tgl. eine Tasse trinken. In schweren Fällen den Tee durch 30 Tr. einer Blutwurztinktur verstärken.

\section{Durchfalltee mit Blutwurz}

- Tormentillae radix, geschnitten 60.0

- Kamillenblüten 20.0

- Gänsefingerkraut 20.0 M.f.spec. ßen, 15 min ziehen lassen, abseihen, bis $3 \times$ tgl. 1 Tasse trinken. Milder Durchfalltee aus getrockneten Heidelbeeren

1 EL getrocknete Heidelbeeren (keine frischen Früchte: die darin enthaltenen Fruchtsäuren würden den Durchfall verstärken) mit $250 \mathrm{ml}$ kaltem Wasser ansetzen, 10 min köcheln, abseihen, mehrmals tgl. 1 Tasse tgl. trinken. Das Kauen von mehrmals tgl. 1 EL getrockneten Heidelbeeren reduziert außerdem analen Juckreiz.

\section{Blutwurztinktur}

30 Tr. der Tinktur auf 1 Tasse Wasser oder Tee, bis zu $3 \times$ tgl. Blutwurztinktur kann als fertige Urtinktur verwendet oder selbst hergestellt werden: $10 \mathrm{~g}$ frische Wurzeln bürsten, zerschneiden, etwas anquetschen (alternativ $5 \mathrm{~g}$ getrocknete Wurzel), in ein Schraubdeckelglas geben, mit $500 \mathrm{ml}$ Doppelkorn aufgießen, verschließen und vier Wochen im Dunkeln stehen lassen, dabei ab und zu schütteln. Anschließend abseihen, in braune Tropfflaschen abfüllen und beschriften.

D.S.: 1 TL der Mischung mit $200 \mathrm{ml}$ siedendem Wasser übergie- 


\section{Fertigarzneimittel}

Gerbstofftees (siehe Kasten) schmecken für viele Patienten sehr unangenehm. Für empfindliche Personen eignen sich daher neben dem milden Tee aus getrockneten Heidelbeeren die Gerbstoffe in Kapselform, insbesondere Tanninalbuminate. Dabei handelt es sich um Verbindungen des Tannin-Gerbstoffs mit einer Eiweißkomponente, die im Darm abgespalten wird. Da der Gerbstoff erst dort freigesetzt wird, reizt er nicht die Magenschleimhaut, zum Beispiel Tannacomp ${ }^{\circledR}$ Tbl. 500 mg oder Tannalbin ${ }^{\circledR}$ Tbl. $500 \mathrm{mg}$ (beide Fa. Medice), bis zu $6 \times$ tgl. 2 Tbl.

Zur Bindung von Flüssigkeit und Reizstoffen eignen sich außerdem Adsorbenzien. Sie sollen in ausreichendem Abstand zu Medikamenten und Mahlzeiten eingenommen werden, zum Beispiel Luvos ${ }^{\circledR}$ Heilerde Kps., $2 \times$ tgl. 3 Kps. (auch als Granulat oder Pulver erhältlich) oder Myrrhinil intest ${ }^{\circledR}$ (Fa. Repha, enthält Kaffeekohle, Myrrhe und Kamillenblüten), $3 \times$ tgl. 4 Tbl.

\section{Antidiarrhoikum vom Opiattyp}

Wenn Reizdarmpatienten wichtige geschäftliche oder gesellschaftliche Termine zu absolvieren haben, verstärkt die Anspannung bereits im Vorfeld die Symptome. Für Durchfallchroniker ist eine Planung solcher Ereignisse einfacher, wenn ein rasch und zuverlässig wirksames Medikament für Notfälle zur Hand ist. Hier kommt besonders Loperamid infrage, zum Beispiel in Imodium akut ${ }^{\circledR}$ (Fa. Johnson \& Johnson). Dabei handelt es sich um einen potenten Peristaltikhemmer, der durch Bindung an Opiatrezeptoren in der Darmwand eine Hemmung der Darmbewegung und eine Verstärkung der Schließmuskulatur des Enddarms bewirkt. Obwohl rezeptfrei erhältlich, sollte das Präparat wegen möglicher Nebenwirkungen nur als Notfallmedikament zur Anwendung kommen. Die maximale Anwendungsdauer beträgt zwei Tage mit einer Tageshöchstdosis von $12 \mathrm{mg}$.

\section{Nachsorge}

Für die probiotische Nachsorge nach Abklingen einer akuten Diarrhö eignet sich Saccharomyces cerevisiae HANSEN CBS 5926-Trockenhefe, zum Beispiel Yomogi ${ }^{\circledR}$ (Fa. Ardeypharm) oder Perenterol ${ }^{\circledR}$ (Fa. Medice), jeweils $2 \times$ tgl. 1 Kps. Diese Hefe hat sich nicht nur in der Nachsorge zur Regenerierung und Stabilisierung der Darmflora bewährt. Auch während der Akutphase von Durchfällen ist eine Gabe von $2 \times \operatorname{tgl} .1 \mathrm{Kps}$. bei manchen Patienten hilfreich. Empfohlen wird diese Dosierung auch zur Prophylaxe von Durchfällen vor einer notwendigen Antibiotikaeinnahme. Hier sollte die Einnahme gleichzeitig mit der Antibiose beginnen und noch bis vier Wochen nach deren Beendigung andauern.

Dieser Artikel ist online zu finden:

http://dx.doi.org/10.1055/a-0617-6884 